\title{
Coronal mass transfer in interbinary loops
}

\author{
J. M. Ferreira ${ }^{1,2}$ and C. A. Mendoza-Briceño ${ }^{3,4}$ \\ 1 Universidade dos Açores, Angra do Heroísmo, Açores, Portugal \\ e-mail: miguelf@mail.angra.uac.pt \\ 2 Centro de Astrofísica da Universidade do Porto, Rua das Estrelas, 4150-762 Porto, Portugal \\ ${ }^{3}$ Centro de Física Fundamental, Facultad de Ciencias, Universidad de Los Andes, Apartado Postal 26, La Hechicera, \\ Merida 5251, Venezuela \\ e-mail: cesar@ula.ve \\ ${ }^{4}$ Space \& Atmosphere Research Center, Department of Applied Mathematics, University of Sheffield, Hicks Building, \\ Hounsfield Road, Sheffield, S3 7RH, England, UK \\ e-mail: c.mendoza@sheffield.ac.uk
}

Received 30 August 2004 / Accepted 14 December 2004

\begin{abstract}
We consider the transfer of coronal mass between the members of a detached binary system along interconnecting rigid magnetic loops. We show that the asymmetry in the gravitational potential drives a flow from the star closer to fill its Roche lobe towards the other star. The loop base pressures, flow velocities and mass fluxes are not externally imposed but are a result of the heating rate along the loop. As the loop heating increases, the base pressures and mass transfer rates increase and the Mach number decreases. If the heating is not uniformly distributed along the loop, but is instead concentrated near one of the stars, then the mass transfer rate either increases or it decreases, and even changes direction, depending on which star the heating is concentrated on. We also discuss the effect of choosing different boundary conditions. Our model predicts mass transfer rates in reasonable agreement with existing observational estimates, but fails to explain the presence of chromospheric material detected between the stars. Additionally, the differential emission measure profile of the loop models is calculated and it is found to be higher in the loop leg close to the secondary star.
\end{abstract}

Key words. stars: activity - stars: magnetic fields - stars: binaries: close - stars: coronae - hydrodynamics

\section{Introduction}

RS CVn systems are detached close binaries with two late-type stars. They are very active systems on which many features of stellar magnetic activity are observed and even enhanced, when compared to single stars. In some of these systems there is evidence suggesting that the interaction between the stellar magnetospheres may originate interbinary flares, interconnecting loops and mass transfer. Observational evidence for interbinary flares is scarce and even somewhat speculative (Simon et al. 1980). Some of the claims for interbinary flares were based on the application of the model by van den Oord (1988). But, according to Ferreira (1998) this model is incorrect, implying that those claims for interbinary flares are invalid (e.g. Doyle et al. 1994; Graffagnino et al. 1995; Pagano et al. 1997). Similarly, evidence for interconnecting magnetic loops channeling mass transfer exists but is inconclusive (Simon et al. 1980; Prés et al. 1995; Buzasi 1991; Pagano et al. 2001; Richards \& Rosolowsky 1998; Busà et al. 1999). Richards \& Rosolowsky (1998) analysed archival IUE spectra of HR 1099 and detected chromospheric emission near the L1 point. Busà et al. (1999) reached analogous conclusions and for the binary AR Lacertae Pagano et al. (2001) found similar evidence. The early theoretical work of Uchida \& Sakurai (1983) supports the view that if the two components of the binary system have considerable magnetic fields, then interconnecting loops are formed.

Recent observational studies show a very dynamical solar corona far different from the traditional static and steady environment (Aschwanden et al. 2001). Theoretical modelling attempts to explain many different kinds of flows such as the Evershed flow in sunspots (e.g. Thomas \& Montesinos 1997), flows in flare and post-flare coronal loops (Craig \& McClymont 1986; Reale et al. 1997; Hori et al. 1997) and flows in activeregion coronal loops (Cargill \& Priest 1980; Mariska \& Boris 1983; Robb \& Cally 1992, hereafter RC92; Patsourakos et al. 2004). In order to model these flows it has been necessary to address fundamental issues such as the nature of the mechanisms that drive the flow and the suitable set of boundary conditions. Of greatest significance is the identification of the loop heating mechanism and how it distributes energy along the loop. A wealth of observational evidence and theoretical modeling indicates that solar coronal heating is concentrated near the chromosphere (e.g. Aschwanden et al. 2001). It is therefore plausible or perhaps even advisable to consider that the same happens in other solar-type stars. 
With the knowledge that flows are so common in the solar corona, it seems reasonable to conclude that if interbinary loops exist then they contain plasma in a dynamical state. In our view, strong evidence for interbinary flares and interbinary flows will require detailed theoretical models capable of producing testable observational predictions. Here we give a first step towards this goal by studying steady flows along interbinary loops. We consider the flow driving mechanism to be either asymmetric gravitational potential or asymmetric heating and we explore the effects of different boundary conditions on the characteristics of the flow. We also discuss the observational implications of our results. This paper is organized as follows: in Sect. 2 we describe the model and its assumptions; in Sect. 3 we present our results, while in Sect. 4 we discuss the implications and observational predictions of our model. Our conclusions are presented in Sect. 5.

\section{The model}

\subsection{The model geometry and assumptions}

The geometry of our model is depicted in Fig. 1. The interbinary loop connects the surfaces of the two spherical stars and has a constant cross-sectional area. We consider that this loop is formed by the interaction between the stellar magnetic fields either in a violent way (e.g. a flare) or by non-violent and low energy release mechanism (e.g. as in the formation of interconnecting loops between solar regions). During, and soon after this interaction, one expects a very fast and dynamical evolution of the newly formed loop. This interesting process will not be considered here due to its complexity, but left for subsequent work. After this phase one expects the plasma in the loop to reach the quasi-steady behaviour that we are modelling in this study.

The physical parameters of the model are those of the well studied RS CVn system HR 1099 (Table 1). This defines the loop length to be $6.3 R_{\odot}$, which is one or two orders of magnitude longer than the typical solar loops studied in the literature (cf. RC92). We assume the magnetic field to be strong enough so that it can be considered as rigid. In this, and generally in any other binary, effective gravity (gravity plus centrifugal force) is not symmetric with respect to the loop midpoint. This is of crucial importance and has significant implications for the flow (cf. Sect. 3). The heating function is assumed to be either a constant per unit volume or concentrated near the most active component of the binary.

\subsection{The equations and boundary conditions}

The equations of mass, momentum and energy conservation along the loop are:

$\rho V=j$

$\rho V \frac{\mathrm{d} V}{\mathrm{~d} s}=-\frac{\mathrm{d} p}{\mathrm{~d} s}+\rho g_{e}$,

$\frac{\mathrm{d}}{\mathrm{d} s}\left(\kappa \frac{\mathrm{d} T}{\mathrm{~d} s}\right)=\left(\rho^{2} Q(T)-H\right)-V\left(\frac{\mathrm{d} p}{\mathrm{~d} s}-5 \frac{k_{\mathrm{B}}}{m_{\mathrm{H}}} \rho \frac{\mathrm{d} T}{\mathrm{~d} s}\right)$

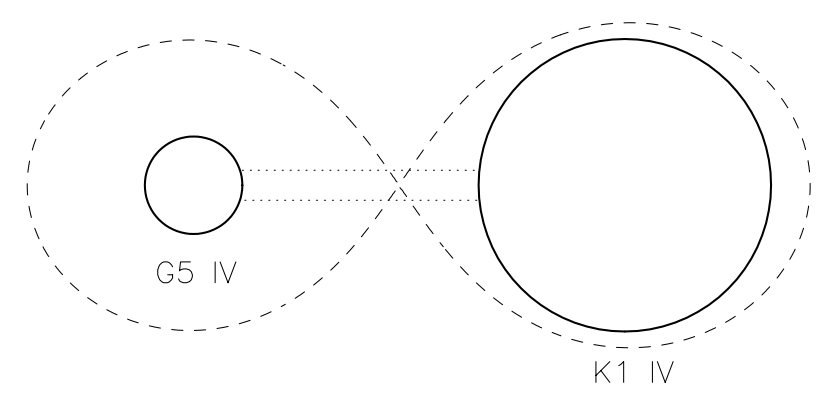

Fig. 1. A schematic representation of the mass transfer model along a straight rigid loop (dotted line) connecting the G5 IV secondary to the KI IV primary. Also shown is the Roche lobe (dashed line)

Table 1. Binary parameters

\begin{tabular}{lccc}
\hline \hline Sp type & K1 IV & G5 V & \\
Radius & $3.9 \pm 0.2$ & $1.3 \pm 0.2$ & $R_{\odot}$ \\
Mass & $1.4 \pm 0.2$ & $1.1 \pm 0.2$ & $M_{\odot}$ \\
$P_{\text {orb }}$ & \multicolumn{4}{c}{$2.83774 \pm 0.00001$} & day \\
\hline
\end{tabular}

where $T$ is the temperature, $p$ is the gas pressure, $s$ is the coordinate along the loop, $V$ is the fluid velocity, $\rho$ is the mass density, $j$ is the mass flux, $H$ is the coronal heating rate, $Q(T)$ is the radiative loss function for optically thin emission, $K \approx 10^{-11} T^{5 / 2}$ is the thermal conductivity parallel to the field and $g_{\mathrm{e}}$ is the effective gravity along the loop. We consider a fully ionized hydrogen plasma so that the equation of state is $P=2 k_{\mathrm{B}} n T$ and $\rho=n m_{\mathrm{H}}$, with $n$ representing the electron number density, $m_{\mathrm{H}}$ the mass of the hydrogen atom and $k_{\mathrm{B}}$ the Boltzmann constant.

The solution of this system of equations requires the specification of four boundary conditions. However, there seems to be no consensus over what the correct boundary conditions should be. The difficulty arises from understanding how to incorporate the effect of the chromosphere and its coupling with the upper atmosphere, without actually introducing and modelling this layer's physics. Some authors argue that one should impose a chromospheric temperature and a pressure at the loop footpoints. It is then the pressure difference between the footpoints that drives the flow (e.g. Cargill \& Priest 1980, RC92). If these are the correct boundary conditions, then one must find a physical mechanism that creates and maintains this pressure difference. Yet, no such mechanism has been suggested.

Another choice is to consider a thermally isolated loop $(\mathrm{d} T / \mathrm{d} s=0$ at the boundaries) as in the works of Serio et al. (1981), Aschwanden et al. (2001) and Noci et al. (1989). With this choice, the base pressures are not imposed but are a result of the equations. Also, a flow only occurs if some sort of asymmetry exists in the system. However, some reserves have also been raised about the correctness of this choice (e.g. Hood \& Anzer 1988). A severe drawback to this choice is that, in general, the solutions obtained cannot be coupled to the chromosphere. At the base, the condition of zero gradient together with the fact that radiative cooling is greater than heating implies that either there is a discontinuity in the heating function or the temperature has a local minimum. Consequently, this choice of boundary condition is not physically well-founded. 
The final set of boundary conditions we shall discuss have for a long time been advocated by McClymont \& Craig (1985a,b). It consists in imposing a zero temperature gradient together with a balance between radiative cooling and heating at the footpoints to correctly represent the chromosphere. As a consequence, the temperature at the footpoints of the loop cannot be imposed.

Although other boundary conditions could be adopted, these three possibilities are the most commonly used in the solar literature. As argued above, the first two possibilities lead to inconsistencies, so the last possibility seems more adequate. However, it is crucial to assess the importance of the boundary conditions in determining the behaviour of the solutions. Therefore, we do not reject a priori any of these boundary conditions, but we study their implications.

\section{Results}

Observations of active RS CVn binaries indicate the presence of coronal loops with high temperature $\left(0.3-3 \times 10^{7} \mathrm{~K}\right)$ and high density $\left(n_{\mathrm{e}} \approx 10^{16} \mathrm{~m}^{-3}\right)$ (e.g. Audard et al. 2001; Ness et al. 2004; Robinson et al. 1996). In the solar contex, the coronal density can vary by a few orders of magnitude, depending whether one is observing quiet region loops, active region loops or even flare loops (e.g. Priest 1982). We should then be cautious and explore the properties of an interbinary loop with different densities and heating rates. To estimate the heating rate, we consider that $H \approx n_{\mathrm{e}}^{2} Q(T)$ so that for a low density quiet region loop as observed on the Sun $n_{\mathrm{e}} \approx 2 \times 10^{14} \mathrm{~m}^{-3}$, and with $Q(T) \approx 10^{-36} \mathrm{~W} \mathrm{~m}^{3}$, we obtain a heating rate $H \approx$ $4 \times 10^{-8} \mathrm{~W} \mathrm{~m}^{-3}$. For a higher density loop $n_{\mathrm{e}} \approx 10^{16} \mathrm{~m}^{-3}$ we have instead $H \approx 10^{-4} \mathrm{~W} \mathrm{~m}^{-3}$.

\subsection{Flow driven by a pressure difference}

Robb \& Cally (1992) have performed a detailed study of siphon flows in hot solar coronal loops driven by a pressure asymmetry between the footpoints. They find that small footpoint pressure asymmetries produce steady subsonic flows, but that the standard picture that large pressure differences invariably lead to supersonic flows is incorrect. Supersonic flows are only possible for high heating, long loops or low pressure. If these conditions are not met, then there are no physical steady state solutions for large pressure asymmetries. Their time-dependent simulations show that in this case a quasi-periodic surge is driven.

Our goal is to see whether these results also apply to large interbinary loops that are not symmetrical with respect to their midpoint, and whether steady state flows in both directions are possible.

The equations are solved integrating from the secondary to the primary. The pressure, temperature and velocity are imposed and the conductive flux at the secondary is iterated until the temperature at the primary reaches the chromospheric value. We start by calculating the static equilibrium which determines the footpoint pressure at the primary surface, $p_{\text {static }}^{\mathrm{p}}$. We then calculate a series of solutions for larger base velocities

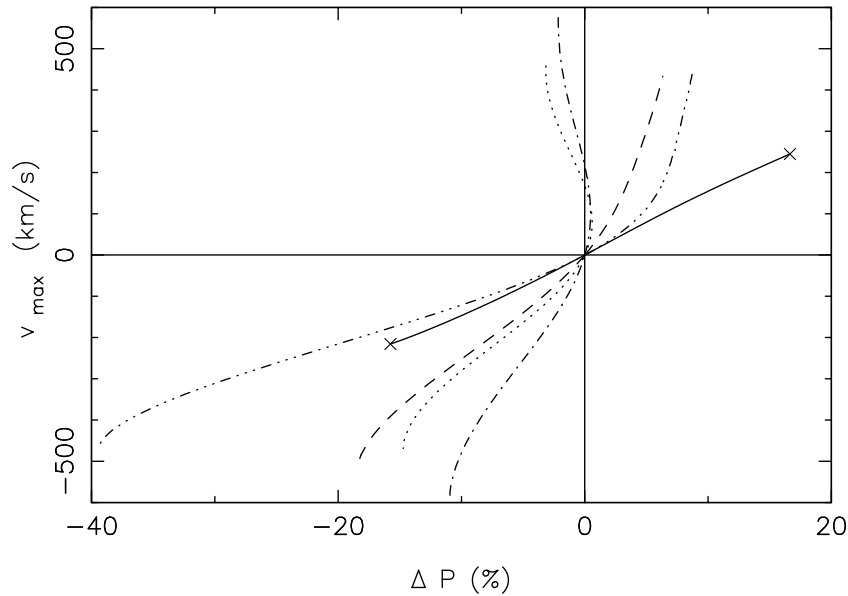

Fig. 2. Maximum velocity along the loop as a function of percentage pressure difference for different values of heating and base pressure at the secondary: $H=10^{-5} \mathrm{~W} \mathrm{~m}^{-3}, p=0.1 \mathrm{~Pa}$, (full line); $H=10^{-5} \mathrm{~W} \mathrm{~m}^{-3}, p=0.05 \mathrm{~Pa}$ (dashed line); $H=10^{-5} \mathrm{~W} \mathrm{~m}^{-3}$, $p=0.025 \mathrm{~Pa}$ (dotted line); $H=2 \times 10^{-5} \mathrm{~W} \mathrm{~m}^{-3}, p=0.1 \mathrm{~Pa}$ (dashedtriple-dotted); $H=5 \times 10^{-5} \mathrm{~W} \mathrm{~m}^{-3}, p=0.1 \mathrm{~Pa}$ (dashed-dotted line). The full line case represents a sequence of subsonic solutions that terminates because the temperature gradient at the downflow footpoint becomes unphysical. All the other curves terminate where the flow becomes supersonic at some point along the loop.

and find the relative pressure difference required to drive the flow that is defined as $\Delta p=\left(p_{\text {static }}^{\mathrm{p}}-p^{\mathrm{p}}\right) / p_{\text {static }}^{\mathrm{p}} \times 100 \%$.

A flow from the secondary to the primary is given by a positive velocity while a negative velocity represents a flow in the opposite direction. Our results are summarized in Fig. 2. On one hand, they are similar to those of RC92. Supersonic flows are possible only if there is high heating or (and) low pressure. Otherwise, for large base velocities there are no solutions, as the temperature gradient near the downflow footpoint becomes of the wrong sign (full line solution in Fig. 2). On the other hand, a new result emerges from this calculations. From the figure, we can see that for low pressure or high heating the same pressure difference can originate two different solutions (dotted and dashed-dotted lines). Furthermore, in some cases these two solutions are for flows in opposite directions! It must be noted that this effect only occurs for flows from the secondary to the primary, i.e., in the opposite direction to what the asymmetry in the effective gravity implies. To support this idea, we have calculated solutions for solar loops with constant solar gravity and asymmetric heating. There is only one steady state solution for flows from the more heated footpoint for a given pressure difference, but for flows from the less heated footpoint there are two solutions for the same pressure difference. Therefore, we conclude that this behaviour results from imposing a flow contrary to what the physics of the loop imposes.

\subsection{Flows in thermally isolated loops}

To determine solutions of thermally isolated loops we impose the temperature, $T_{b}=2 \times 10^{4} \mathrm{~K}$, and its gradient, $\mathrm{d} T / \mathrm{d} s=0$ at the secondary footpoint and iterate the gas pressure and the fluid velocity until these same boundary conditions are obeyed 


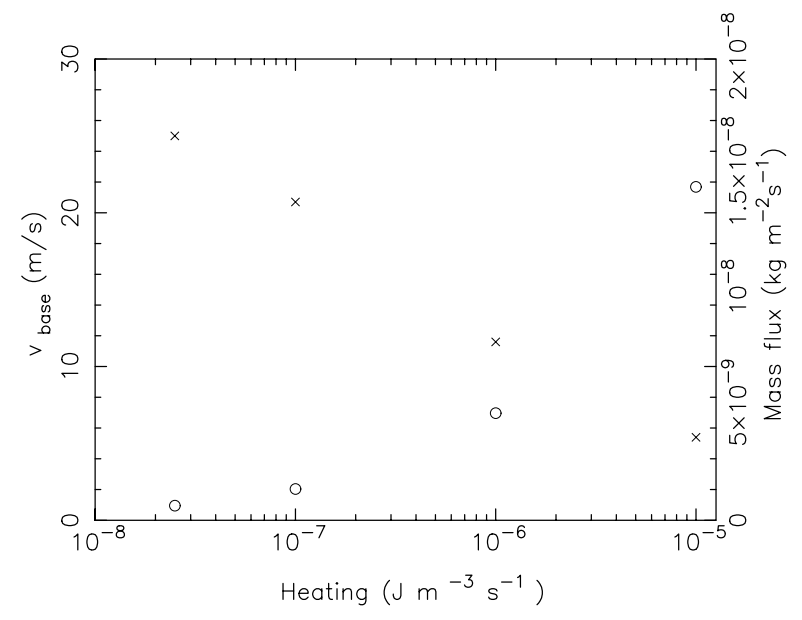

Fig. 3. Absolute values of base velocity (crosses) and mass flux (open circles) for different values of the heating rate. As the heating decreases, the base velocity raises but the mass flux decreases. The loop peak temperatures in order of increasing heating are: $2.5 \mathrm{MK}, 3.6 \mathrm{MK}$, 6.8 MK and 12.9 MK. The base pressure at the secondary in order of increasing heating are: $0.0071 \mathrm{~Pa}, 0.022 \mathrm{~Pa}, 0.13 \mathrm{~Pa}, 0.89 \mathrm{~Pa}$. Below $H \approx 2 \times 10^{-8} \mathrm{~W} \mathrm{~m}^{-3}$ the flow becomes supersonic at some point along the loop.

at the other footpoint. Contrary to what we find in the previous section, here there is only one possible solution and the flow is invariably from the primary towards the secondary. Certainly, this is what one would expect as the primary fills a large fraction of its Roche lobe. As we decrease the heating rate, the footpoint pressures decrease, the maximum Mach number along the loop increases but the mass transfer rate decreases. This is shown in Fig. 3. Also, it is important to remark that the base pressure and coronal heating are not set independently of each other. Therefore, steady state solutions with low heating and high pressure, or high heating and low pressure, do not exist within this framework. In comparison with the previous boundary conditions, here we find that large mass fluxes imply high heating and high pressure with very subsonic velocities.

The assumption of uniform heating is a very strict constraint hardly to be met in a real loop. First, as described earlier, it is likely that the heating is concentrated near the footpoints of the loop. Second, as the loop footpoints are on the surfaces of two different stars one expects the heating to be more concentrated near the most active star. In the particular case of the HR 1099 binary, the primary component is significantly more active magnetically than the secondary (e.g. Zhai $\&$ Zhang 1996). Let us consider a non-uniform heating function that reflects the concentration of the heating near one of the footpoints. We choose

$H=H_{0}+H_{1} \exp \left[-\frac{\left(d-R_{1}-s\right)}{s_{H}}\right]$

if the heating is concentrated near the primary and

$H=H_{0}+H_{1} \exp \left[-\frac{\left(s-R_{2}\right)}{s_{H}}\right]$

if the heating is concentrated near the secondary. Here, $H_{0}$ represents a small uniform heating that we consider to be

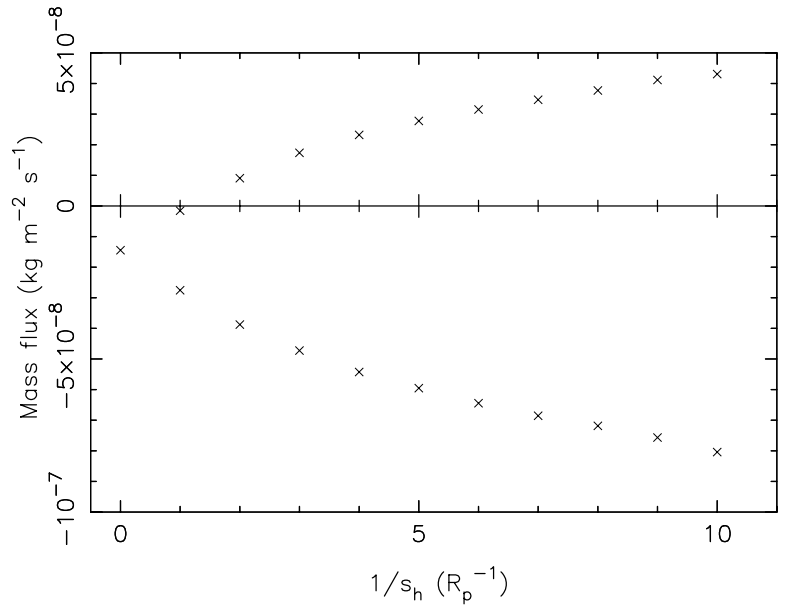

Fig. 4. Mass flux as a function of heating asymmetry. All solutions have the same total heating. The upper part is for solutions with heating concentrated near the secondary and the lower part for solutions with heating concentrated near the primary.

$H_{0}=10^{-7} \mathrm{~W} \mathrm{~m}^{-3}$. The parameter $s_{H}$ is the spatial scale-length of the variable heating and the value of $H_{1}$ is chosen so that the total heating, $H_{t}=\int H(s) \mathrm{d} s$, is the same as in the uniform heating case. The asymmetry in the energy deposition rate evaporates material from the more heated side of the loop and pushes it towards the other side of the loop. Therefore, if the heating is concentrated near the primary, it enhances the effect of the gravity asymmetry and increases the mass flux. On the other hand, if the heating is concentrated near the secondary, its effect is contrary to that of gravity and leads to a decrease in the mass flux or, if the heating asymmetry is significant, reverses the direction of the mass flow (Fig. 4). For very high heating asymmetries (not represented in this plot) there are no solutions with a single local temperature maximum. Instead, the solutions are characterized by a temperature inversion along the loop. At this minimum, the temperature never decreased below coronal values for all the cases considered. In Fig. 5 we plot the velocity, pressure and temperature of a loop with uniform heating, heating concentrated near the primary and heating concentrated near the secondary. It is evident from the figure that loops with asymmetric heating have smaller peak temperatures than uniformly heated loops. For heating concentrated near the primary, the pressure asymmetry between the footpoints increases as a result of an increase in the pressure at the primary and a decrease of the pressure in the secondary. On the other hand, a loop with heating concentrated near the secondary has both footpoints with a lower pressure than the uniformly heated loop. The mass flux is very sensitive to the spatial distribution of heating while the temperature has a smaller dependence.

\subsection{Flows in loops with energy balance between heating and radiation in the chromosphere}

Here we study the effect of imposing an energy balance between heating and cooling together with a zero temperature gradient at the base. The temperature at the base cannot be 

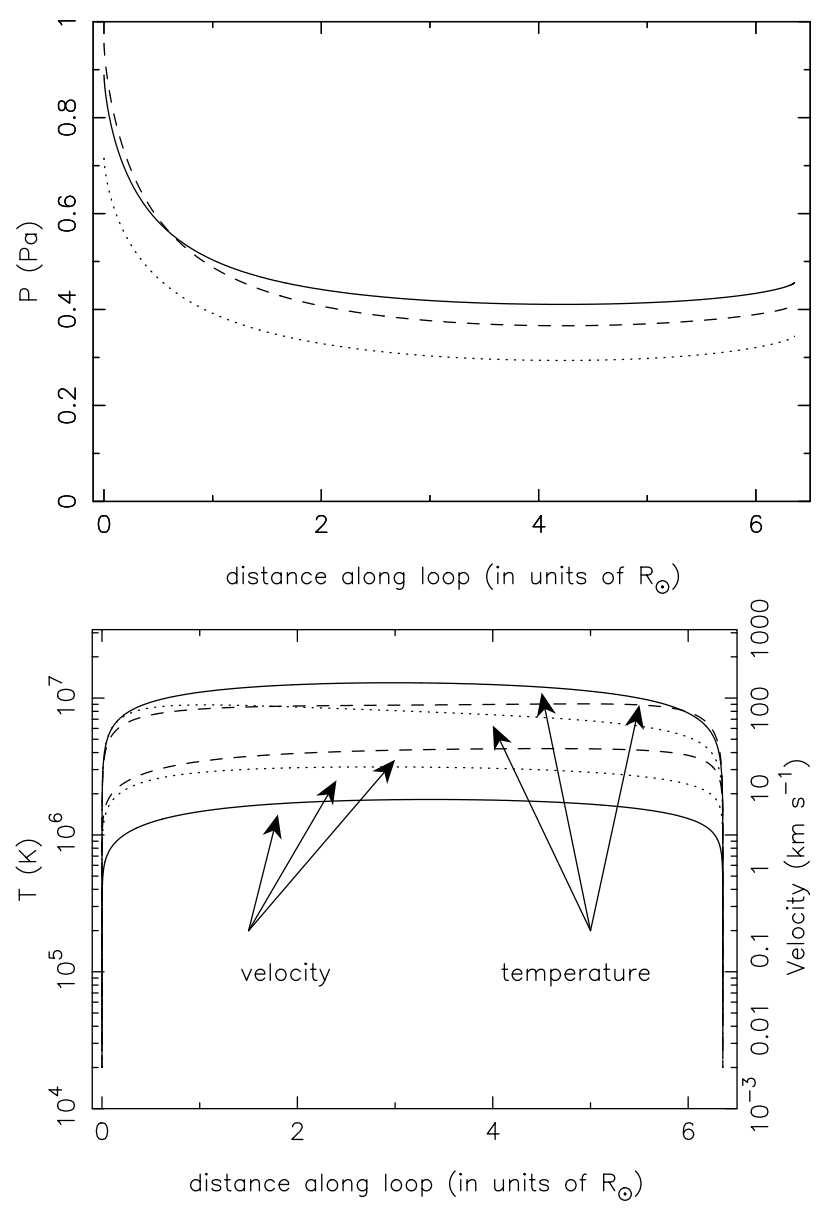

Fig. 5. Pressure (upper panel), temperature and velocity (lower panel) along the loop for different heating distributions but same total heating. Represented are the uniform heating case (full line), and the spatial heating cases for $s_{H}=R_{p} / 10$ (dashed lines - heating concentrated near the primary star; dotted line - heating concentrated near the secondary star).

imposed but is a result of the computation. In order to keep this temperature at around $2 \times 10^{4} \mathrm{~K}$ and mimic the effect of the chromosphere, we change the cooling function such that the cooling decreases continuously to zero between $1.95 \times 10^{4}<$ $T<2 \times 10^{4} \mathrm{~K}$. This is commonly done in time-dependent simulations (e.g. Spadaro et al. 2003). By comparison, in the solutions computed in the previous section the cooling is several orders of magnitude larger than the heating at the base. Therefore, for similar heating rates, the cooling in this case has to decrease substantially to obey the boundary condition. This is achieved at temperatures close to $1.95 \times 10^{4} \mathrm{~K}$.

We can write the energy equation in the more elucidative form

$$
\begin{aligned}
\frac{\mathrm{d}}{\mathrm{d} s}\left(\kappa \frac{\mathrm{d} T}{\mathrm{~d} s}\right)= & \left(\rho^{2} Q(T)-H\right) \\
& -j\left(g_{\mathrm{eff}}-\frac{\mathrm{d}}{\mathrm{d} s} \frac{V^{2}}{2}-5 \frac{k_{\mathrm{B}}}{m_{\mathrm{H}}} \frac{\mathrm{d} T}{\mathrm{~d} s}\right) .
\end{aligned}
$$

This way the enthalpy, kinetic and gravitational contributions to the energy balance become clear. At the base, the kinetic and the gravitational energy fluxes contribute as energy sources in

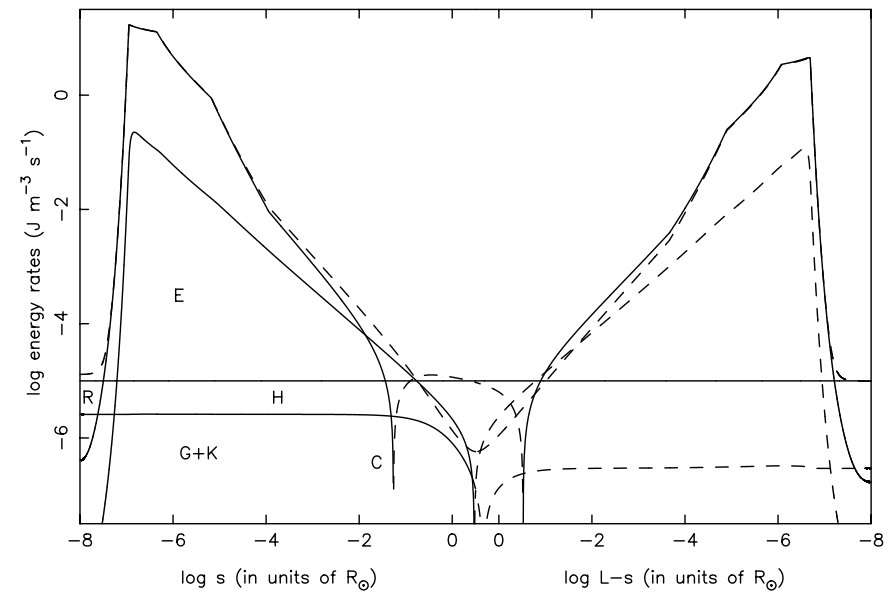

Fig. 6. Energy sources (solid lines) and energy sinks (dashed lines) along a loop with uniform heating and a flow driven by the gravitational potential asymmetry. Shown are: radiation $(\mathrm{R})$, heating $(\mathrm{H})$, conduction (C), enthalpy (E) and gravitational and kinetic energy $(\mathrm{G}+\mathrm{K})$. Notice that the variables are plotted against the logarithm of the distance from each footpoint.

the downflow leg and as energy sinks in the upflow leg, while the enthalpy has no contribution.

We solve the equations subject to these boundary conditions for a loop with constant heating rate $H=10^{-5} \mathrm{~W} \mathrm{~m}^{-3}$. The results are very similar to what we find in the case of a fixed base temperature and zero flux (cf. Sect. 3.2). The base pressures and maximum loop temperature are virtually identical in both cases. In the present case, the base temperatures are very close to $1.95 \times 10^{4} \mathrm{~K}$ in comparison with $2 \times 10^{4} \mathrm{~K}$ for the thermally isolated loops implying, due to mass conservation, similar differences in the base velocity, $V_{b}=5.27 \mathrm{~m} / \mathrm{s}$ and $V_{b}=5.40 \mathrm{~m} / \mathrm{s}$ respectively. The role of the different energy sources in the solution is shown in detail in Fig. 6. Here solid line represent energy sources and dashed lines energy sinks. There is a balance between heating and radiation in the chromosphere and a balance between conduction and radiation in the transition region.

We conclude that, in what concerns equilibrium, these boundary conditions give the same result as that of a thermally isolated loop. Therefore, all the results found in the previous section apply here as well. In spite of the fact that this set of boundary conditions represents more correctly the interaction with the chromosphere, it is numerically harder to implement, so it is justified to consider thermally isolated loops. This does not imply that the stability of the solutions is independent of the boundary condition. On the contrary, the solutions' stability is known to be highly dependent on the choice of the boundary condition (e.g. McClymont \& Craig 1985a)

\section{Discussion}

\subsection{The importance of the boundary conditions}

It is extremely complicated to model the interaction between coronal loops and the deep solar atmosphere accurately. Therefore, mathematical conditions are imposed at a boundary that are believed to mimic this complicated interaction. 
Different choices of boundary conditions have been proposed to address the problem of flows along coronal loops.

If the gas pressure at this boundary is imposed from below, then, as shown in Sect. 3.1, different base pressures at the loop footpoints can drive subsonic and supersonic flows in either direction. Supersonic flows occur only with high heating rate or low gas pressure. The asymmetry in the gravitational potential does not disallow flows to occur in both directions. But in loops with high heating or low pressure there are no steady state solutions for base pressure differences imposing a flow contrary to what the gravitational asymmetry enforces.

Radically different results are found if one chooses other sets of boundary conditions. Two cases have been considered. Those representing thermally isolated loops and those representing loops with balance between heating and radiation at the base. Both these cases give identical results and the flow is driven by asymmetries in the heating or cooling along the loop. The base pressures and base velocities are not imposed but are a consequence of the equations.

In our perspective, fixing the pressure at the boundary gives erroneous results, as it is based on an unknown mechanism capable of maintaining the pressure at the base, independently of the loop characteristics (length, heating rate, ...). Therefore, in what follows we shall only discuss the observational implications of the results obtained with the set of boundary conditions presented in Sect. 3.2.

\subsection{Observational predictions}

This model predicts the existence of a flow along a uniformly heated interbinary loop from the star that fills most of its Roche lobe to the other star, driven by the gravitational potential asymmetry. The velocity of this flow increases as the heating decreases and supersonic flows only occur with very low heating rates. Interestingly, the larger the velocity at the base, the lower are the base pressure and density. So, high mass transfer rates require large heating rates with low velocities. The velocities obtained with this model are significantly below $1 \mathrm{~km} \mathrm{~s}^{-1}$ at chromospheric temperatures, implying that the Doppler effect due to such flows is undetectable.

The present model is not capable of explaining the detection of chromospheric material in between the binaries (Busà et al. 1999). This cool plasma could be in quasi-static equilibrium and represent a prominence seen in emission (Buzasi et al. 1991), or it could be in a dynamical state and be the result of cool post-flare loops. Our model will be extended to include time-dependent variations and address in detail the later possibility. It may also be able to explain the significant velocities at chromospheric temperatures that have been detected in some binary systems (e.g. Simon et al. 1980) that have been attributed to interbinary mass transfer. However, it may prove difficult to reconcile the persistent of this chromospheric material at different epochs and in different binary systems with transient phenomena like flares ${ }^{1}$.

As the X-ray emission from RS CVn binaries is very often dominated by one of its components, we investigated the effect

\footnotetext{
1 We owe this comment to the referee of the paper.
}

of a spatial dependent heating rate. We find that this can increase the mass transfer rates significantly, but it can also have the effect of reversing the direction of the flow. This occurs if the heating asymmetry is significant to oppose the effect of gravity.

An estimate of the mass transfer rate by this process is

$\frac{\mathrm{d} M}{\mathrm{~d} t}=1.8 \times 10^{-11} f_{1} f_{2} \quad M_{\odot} \mathrm{year}^{-1}$

where $f_{1}$ is the fraction of the primary stellar disk occupied by the interbinary loop, $f_{2}$ the fraction of time in a year in which there is an interbinary loop and we have considered a mass flux of $5 \times 10^{-8} \mathrm{~kg} \mathrm{~m}^{-2} \mathrm{~s}^{-1}$ (cf. Fig. 4). For this to be comparable with the value of mass exchange rate given by Buzasi et al. (1991) of $10^{-12} M_{\odot}$ year $^{-1}$ it requires a significant fraction of the stellar surface to be connected to its binary component during a substantial fraction of the time. Higher mass fluxes are likely to occur during time dependent flare events with detectable observational signatures. But it is unlikely that this flows can be sustained during considerable periods of time. Another possibility is that of high mass fluxes along loops with temperature inversions caused by localized heating near the loop base. These type of solutions have been studied in stellar loops on rapidly rotating single stars by Ferreira \& MendozaBriceño (1997) and in solar loops by Patsourakos et al. (2004). We have conducted a preliminary study of these solutions in the context of interbinary coronal mass transfer models. We have considered several heating functions and although higher mass fluxes were found, they are of the same order of magnitude as the ones shown here. Also, we were unable to find solutions in which the temperature inversion reached chromospheric like temperatures. However, a more detailed study is required to address this possibility.

If it were possible to observe an interbinary coronal loop of the kind modelled here, it would be dominated by the contribution of the section of the loop closer to the secondary star. This is evident from the differential emission measure calculated for the solutions obtained in Sect. 3.2 (Fig. 7). Due to the asymmetry in the gravitational potential, the portion of the loop closer to the secondary is at a significantly higher gas pressure than the portion near the primary, resulting in a higher differential emission measure.

\section{Conclusions}

RS CVn systems are magnetically active detached binaries for which mass transfer is believed to occur, either continuously or sporadically. We have considered the steady-state mass transfer between the two stellar components along an interconnecting rigid loop of constant cross-sectional area.

The effect of imposing different boundary conditions was analysed in detail. We have argued that fixing the base pressure gives inadequate results. Physically correct results are obtained when either isolated loops or loops with an energy balance between heating and radiative cooling at the base are considered.

The form of the gravitational potential introduces an asymmetry in the energy equation that induces a flow from the primary to the secondary star. Large mass transfer rates are driven 


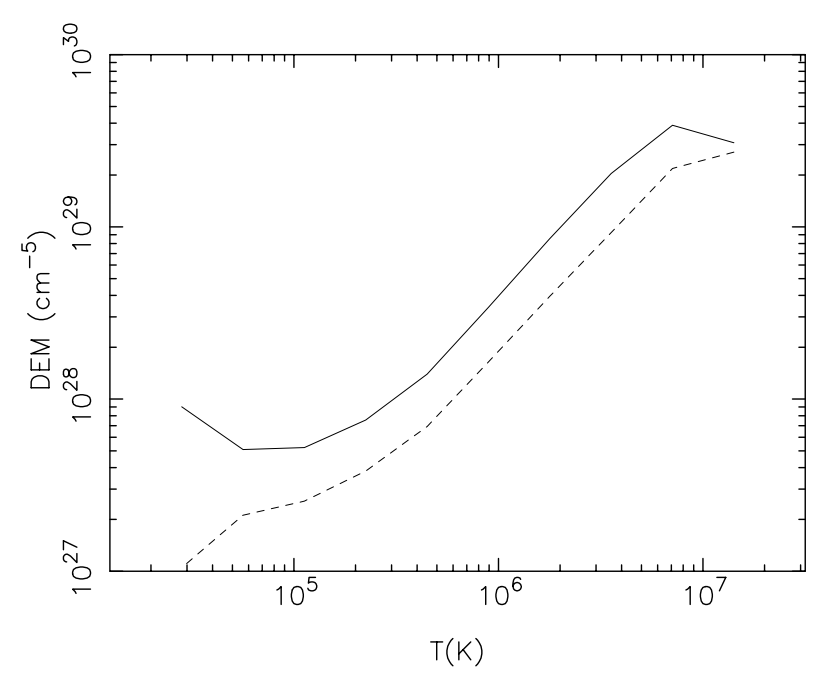

Fig. 7. Differential emission measure in intervals of $\log T=0.3$ of a uniformly heated loop with $H=10^{-5} \mathrm{~W} \mathrm{~m}^{-3}$. The contribution of the half-loop close to the primary is represented by the dashed line and the other half is presented by the full line.

by high heating localized near the footpoints of the primary. However, if heating is concentrated near the secondary, then the mass transfer occurs in the opposite sense, from the secondary to the primary.

The values of mass transfer rates resulting from the model are in reasonable agreement with existing observational estimates (Buzasi et al. 1991). Yet, the model fails to explain the observation of chromospheric material in between the stars (e.g. Busà et al. 1999). A loop with time-variable heating will be investigated in the future as a possible explanation to this observations.

Acknowledgements. This work was initiated during a visit by J.M.F. to the Universidad de los Andes and it is a pleasure to acknowledge the kind hospitality offered. We would like to thank the referee for pointing out to us relevant references where the presence of chromospheric material near the L1 point of RS CVn binaries is established. We are grateful to Vítor Costa for some elucidative discussions on the physics of differential emission measure and to Teresa Marques for carefully reading of the manuscript. C.A.M.-B. would like to thank the CDCHT of the Universidad de los Andes for financial support. This work was partially supported by grants POCTI/1999/FIS/34549 and POCTI/CFE-AST/55691/2004 approved by FCT and POCTI, with funds from the European Community program FEDER.

\section{References}

Aschwanden, M. J., Schrijver, C. J., \& Alexander, D. 2001, ApJ, 550, 1036

Audard, M., \& Güdel, M., Sres, A. 2001, in Abst. Symp., Two years of Science with Chandra, Washington, 102

Busà, I., Pagano, I., Rodonò, M., Neff, J. E., \& Lanzafame, A. C. 1999, A\&A, 350, 571

Buzasi, D. L., Huemenoerder, D. P., \& Ramsey, L. W. 1991, PASP, 103, 1077

Cargill, P. J., \& Priest, E. R. 1980, SoPh, 65, 251

Craig, I. J. D., \& McClymont, A. N. 1986, ApJ, 307, 367

Doyle, J. G., Mitrou, C. K., Mathioudakis, M., \& Antonopoulos E. 1994, A\&A, 283,522

Ferreira, J. M. 1998, A\&A, 335, 248

Ferreira, J. M., \& Mendoza-Briceño, C. A. 1997, A\&A, 327, 252

Graffagnino, U. G., Wonnacott, D., \& Schaeidt, S. 1995, MNRAS, 275,129

Hood, A., \& Anzer, U. 1988, SoPh, 115, 61

Hori, K., Yokoyama, T., Kosugi, T., \& Shibata, K. 1997, ApJ, 489, 426

Mariska, J. T., \& Boris, J. P. 1983, ApJ, 267, 409

McClymont, A. N., \& Craig, I. J. P. 1985a, ApJ, 289, 820

McClymont, A. N., \& Craig, I. J. P. 1985b, ApJ, 289, 834

Montesinos, B., \& Thomas, J. H. 1997, Nature, 390, 485

Ness, J.-U., Güdel, M., Schmitt, J. H. M. M., et al. 2004, A\&A, 427, 667

Noci, G., Spadaro, D., Zappala, R. A., \& Antiochos, S. K. 1989, ApJ, 338,1131

Pagano, I., Ventura, R., Rodonò, Peres, O., \& Micela, G. 1997, A\&A, 318, 467

Pagano, I., Rodonò, M., Linsky, J. L., et al. 2001, A\&A, 365, 128

Patsourakos, S., Klimchuk, J. A., \& MacNeice, P. J. 2004, ApJ, 603, 322

Prés, P., Siarkowski, M., \& Sylwester, J. 1995, MNRAS, 275, 43

Reale, F., Betta, R., Peres, G., \& Serio, S. 1997, A\&A, 325, 782

Richards, M. T., \& Rosolowsky, E. W. 1998, ASP Conf. Ser. 154, ed. R. A. Donahue, \& J. A. Bookbinder, CD-2038

Robb, T. P., \& Cally, P. S. 1992, ApJ, 397, 329

Robinson, R. D., Airapetian, V. S., Maran, S. P., \& Carpenter, K. G. 1996, ApJ, 469, 872

Serio, S., Peres, G., Vaiana, G. S., et al. 1981, ApJ, 243, 288

Simon, T., Linsky, J. L., \& Schiffer, F. H. 1980, ApJ, 241, 759

Spadaro, D., Lanza, A. F., Lanzafame, A. Z., et al. 2003, ApJ, 582, 486

Uchida, Y., \& Sakurai, T. 1983 in Activity in Red Dwarf Stars, ed. P. Byrne, \& M. Rodonò (Dordrecht: D. Reidel), 629

van den Oord, G. H. J. 1988, A\&A, 205, 167

Zhai, D. S., \& Zhang, X. B. 1996, A\&A, 309, 530 